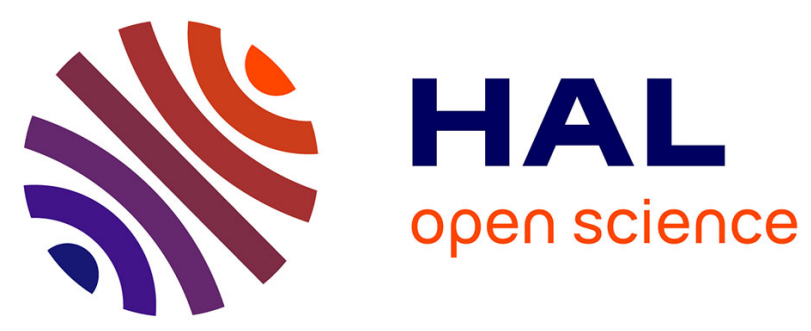

\title{
Palaeoenvironments and prehistoric interactions in northern France from the Eemian Interglacial to the end of the Weichselian Middle Pleniglacial
}

\author{
Jean-luc Locht, Emilie Goval, Pierre Antoine, Sylvie Coutard, Patrick
}

Auguste, Clément Paris, David Hérisson

\section{To cite this version:}

Jean-luc Locht, Emilie Goval, Pierre Antoine, Sylvie Coutard, Patrick Auguste, et al.. Palaeoenvironments and prehistoric interactions in northern France from the Eemian Interglacial to the end of the Weichselian Middle Pleniglacial. Frederick W. F. Foulds; Helen C. Drinkall; Angela R. Perri; David T. G. Clinnick. Wild Things: Recent advances in Palaeolithic and Mesolithic research, Oxbow Books, pp.70-78, 2014, 978-1782977469. hal-02335790

\author{
HAL Id: hal-02335790 \\ https://hal.science/hal-02335790
}

Submitted on 12 Dec 2019

HAL is a multi-disciplinary open access archive for the deposit and dissemination of scientific research documents, whether they are published or not. The documents may come from teaching and research institutions in France or abroad, or from public or private research centers.
L'archive ouverte pluridisciplinaire HAL, est destinée au dépôt et à la diffusion de documents scientifiques de niveau recherche, publiés ou non, émanant des établissements d'enseignement et de recherche français ou étrangers, des laboratoires publics ou privés. 


\title{
An offprint from Wild Things \\ Recent Advances in Palaeolithic and Mesolithic Research
}

\author{
Edited by \\ Frederick W. F. Foulds, Helen C. Drinkall, Angela R. Perri, \\ David T. G. Clinnick and James W. P. Walker
}

Hardcover Edition: ISBN 978-1-78297-746-9

Digital Edition: ISBN 978-1-78297-747-6

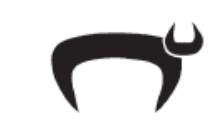

Oxbow Books

Oxford \& Philadelphia 
Published in the United Kingdom in 2014 by

OXBOW BOOKS

10 Hythe Bridge Street, Oxford OX1 2EW

and in the United States by

OXBOW BOOKS

908 Darby Road, Havertown, PA 19083

(C) Oxbow Books and the authors 2014

Hardcover Edition: ISBN 978-1-78297-746-9

Digital Edition: ISBN 978-1-78297-747-6

A CIP record for this book is available from the British Library

All rights reserved. No part of this book may be reproduced or transmitted in any form or by any means, electronic or mechanical including photocopying, recording or by any information storage and retrieval system, without permission from the publisher in writing.

Printed in the United Kingdom by Hobbs the Printers, Totton, Hampshire

For a complete list of Oxbow titles, please contact:

\section{UNITED KINGDOM}

Oxbow Books

Telephone (01865) 241249, Fax (01865) 794449

Email: oxbow@oxbowbooks.com

www.oxbowbooks.com

\author{
UNITED STATES OF AMERICA \\ Oxbow Books \\ Telephone (800) 791-9354, Fax (610) 853-9146 \\ Email: queries@casemateacademic.com \\ www.casemateacademic.com/oxbow
}

Oxbow Books is part of the Casemate group

Front cover: Wild Things conference logo. (c) Kloe Rumsey, 2011.

Back cover: The entrance to Cathole Cave. (C) George Nash. 


\section{Contents}

Preface $\mathrm{V}$

List of Contributors vi

1. Chronology of the Mid Upper Palaeolithic of European Russia: Problems and prospects 1 Natasha Reynolds

2. Invisible Individuals, Visible Groups: On the evidence for individuals and groups at the Lower Palaeolithic site of Caddington, Bedfordshire, UK . 12 Frederick W. F. Foulds

3. Alpine 'Hunters' from the Middle Mesolithic to Early Neolithic: A contribution to the study of lithic industries from two high altitude loci (Gerland and La Mare) in Vercors, Northern French Alps

Alexandre Angelin

4. The Phenomenon of Mesolithic Settlement within the Bohemian Paradise Area,

Czech Republic 61

Petr Šida, Marta Moravcová, Dagmar Vokounová Franzeová and Jan Prostředník

5. Palaeoenvironments and Prehistoric Interactions in Northern France from the Eemian Interglacial to the End of the Weichselian Middle Pleniglacial Jean-Luc Locht, Emilie Goval, Pierre Antoine, Sylvie Coutard, Patrick Auguste, Clément Paris and David Hérisson

6. The Feasibility of Reconstructing Neanderthal Demography as an Approach to Examining Extinction

Danae Rebecca Dodge

7. Le Cuzoul De Gramat (Lot, France): A key sequence for the early Holocene in southwest France

Nicolas Valdeyron, Aureade Henry, Benjamin Marquebielle, Bruno Bosc-Zanardo, Bernard Gassin, Sylvene Michel and Sylvie Philibert

8. Human Craniometric Variation Supports Discontinuity at the Late Glacial Maximum in Europe 106 Ciarán Brewster, Ron Pinhasi and Christopher Meiklejohn 
9. Funerary Contexts: The case study of the Mesolithic shellmiddens of Muge (Portugal) 119

Olívia Figueiredo, João Cascalheira, João Marreiros, Telmo Pereira, Cláudia Umbelino and Nuno Bicho

10. Fire as a Component of Mesolithic Funerary Rituals: Charcoal analyses from a burial in Cabeço da Amoreira (Muge, Portugal) 126 Patrícia Diogo Monteiro, João Cascalheira, João Marreiros, Telmo Pereira and Nuno Bicho

11. Animal Magic: The discovery of Upper Palaeolithic Parietal art in Cathole Cave, Gower Peninsula, South Wales George Nash

12. Ideology of the Hunt and the End of the Epi-Palaeolithic 144

Piotr Jacobsson

13. Animal Exploitation Strategies in Eastern Aquitaine (France) during the Last Glacial Maximum 160

Jean-Christophe Castel, Myriam Boudadi-Maligne, Sylvain Ducasse, Caroline Renard, François-Xavier Chauvière, Delphine Kuntz and Jean-Baptiste Mallye

14. Locating Potential Mesolithic Fish Sites in Britain using Predictive Modelling:

Applying the 'fishing site model' to British conditions 175 Kris Hall

15. Foragers and Farmers in Mesolithic/Neolithic Europe, 5500-3900 cal. BC:

Beyond the anthropological comfort zone 185 Peter Rowley-Conwy 


\title{
5. Palaeoenvironments and Prehistoric Interactions in Northern France from the Eemian Interglacial to the End of the Weichselian Middle Pleniglacial
}

\author{
Jean-Luc Locht, Emilie Goval, Pierre Antoine, Sylvie Coutard, \\ Patrick Auguste, Clément Paris and David Hérisson
}

\begin{abstract}
The Upper Pleistocene in the north of France is characterised by discontinuous human presence, closely linked to climatic fluctuations. Human groups were mainly present during the Eemian interglacial and at the beginning of the Weichselian glacial period, as well as during the Late Glacial, in correlation with large mammal populations. Upper Pleistocene climatic fluctuations impacted on raw material access, which partly conditioned lithic production modalities. However, the influence of cultural traditions remains important, both for lithic production and subsistence strategies.
\end{abstract}

\section{Introduction}

The excavation of abundant Middle Palaeolithic sites over the past twenty years has provided invaluable chronological and environmental information on Upper Pleistocene human occupation in the north of France. This region is considered here to extend from the Seine to the Escaut Basins (Figure 5.1).

The study discussed in this paper is based on data from 47 excavated archaeological levels, representing a total surface area of about $55,952 \mathrm{~m}^{2}$ (Table 5.1), combined with a unique frame of reference in Europe for Pleistocene sequences in loessic context. A total of 75,612 artefacts have been unearthed in these levels. From a chronological viewpoint, five of these levels are contemporary with the Eemian interglacial (Marine Isotope Stage 5e, see Table 5.1 and Figure 5.2), seven are attributed to MIS 5c, ten to MIS 5a and eight others, occurring in steppic soils, to the end of MIS 5a. Lastly, thirteen are contemporary with the end of the Lower Pleniglacial (MIS 4) or Middle Pleniglacial (MIS 3). This significant mass of information has enabled us to analyse interactions between climatic fluctuations, responsible for palaeoenvironmental modifications, and prehistoric occupations. Here we focus on four main themes: occupation density, raw materials, lithic industries and faunal remains.

\section{Climatic changes and human occupations}

During the Eemian, human occupations only appear to be preserved in fluviatile sediments (for example, at Caours - see Locht et al. 2010). At present, these are rare for taphonomic reasons (interglacial soil erosion from the first climatic deterioration at the beginning of the Glacial period onwards) (Locht 2004; Roebroeks and Speelers 2002), and do not expose a realistic record of the Neanderthal occupation of our regions during this interglacial (Figure 5.3). The climate and environment is comparable to that of the present temperate zone, with oceanic influence and deciduous woodlands. The forested landscape was closed, with abundant animal species represented by few specimens.

A slow climatic degradation and a progressive continentalisation of the climate in successive stages is observed from the beginning of the Weichselian glacial period (MIS 5d to 5a) (Antoine et al. 1994, 2003, 2011a, b). The faunal spectrum is less varied, but sizeable herds of herbivores were clearly present. 


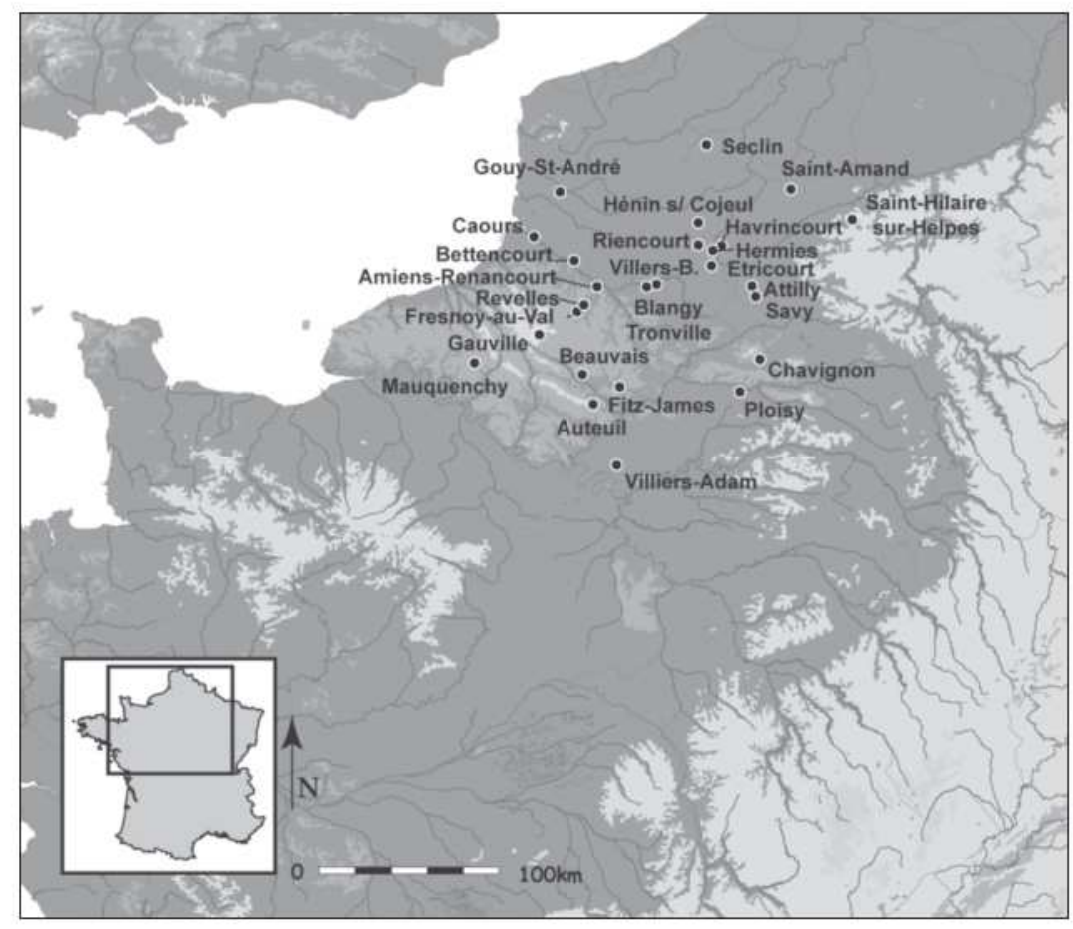

Figure 5.1. Location of the sites.

Sites are preserved in colluvial plateau sediments and especially on lower slopes where pedosedimentary activity is most developed. The high number of well-preserved sites due to favourable conditions seems to indicate that this type of environment was propitious to the implantation and subsistence of Middle Palaeolithic hunting groups.

During the Lower Weichselian glacial maximum (MIS 4), northern Europe seems to be abandoned by human populations. The recolonisation of these regions begins at the end of MIS 4 (Locht 2004) and continues during the Middle Pleniglacial (MIS 3). Again, sites are preserved in loess sediments on slopes and plateaus. The climate, environment and animal communities are globally characteristic of 'Mammoth steppe' conditions (Guthrie 1982), but human occupation appears to occur during short phases of climate improvement (interstadials), during which one can note the formation of Boreal brown soil type horizons. As there are fewer sites for this period than during the early glacial Weichselian, it is probable that human occupation was less dense at this time.

At the end of the Middle Pleniglacial, the first Early Upper Palaeolithic occupations appear in the north of France. Thus, the Havrincourt human occupations (Sector 2, Level 1: early Gravettian) and AmiensRenancourt 2 in the suburbs of Amiens (early Gravettian) occur at about 28,500 BP (around $34 \mathrm{ka} \mathrm{cal}$. BP), in a phase of relative climatic improvement during MIS 3 (Goval and Hérisson 2012; Paris et al. 2013).

\section{Climate changes and raw materials}

Apart from a few rare exceptions, the lithic series are made in Cretaceous flint issued from chalk, available throughout the region. Raw material access appeared difficult during the Eemian, given that temperate forests were abundant that must have concealed flint outcrops. Raw materials, therefore, must only have been accessible at tree falls or riverbeds. The five occupation levels at Caours indicate that flint blocks of reduced dimensions were collected from coarse alluvial deposits in the nearby Scardon. An identical situation occurred during the early Weichselian glacial, characterised by a continental environment with a Boreal forest and open herbaceous areas (Antoine et al. 1994). The abundant lithic series contemporary with this slow phase of climatic degradation (MIS $5 d$ to $5 a$ ) also show that smaller blocks, gathered from broken stones on the plateau, were selected for reduction.

When the Lower Pleniglacial climatic deterioration eroded the chalky slopes, good-quality flint nodules of large size became available. This period corresponds to recurring Mousterian occupations at the base of these chalk taluses at the end of MIS 4 (for example Fitz-James, Teheux, 2001; Ault, Antoine and Auguste, 


\begin{tabular}{|c|c|c|c|c|c|c|c|c|c|c|c|c|c|c|c|c|c|c|c|c|c|c|c|c|c|}
\hline שֶّ & 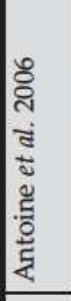 & 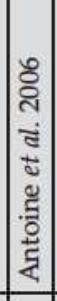 & 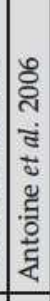 & 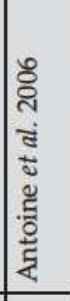 & 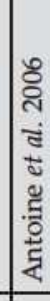 & 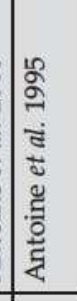 & 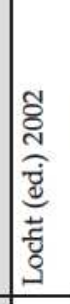 & 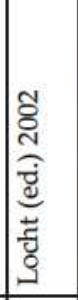 & 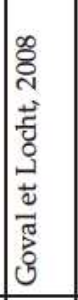 & 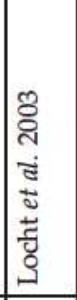 & 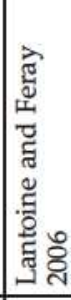 & 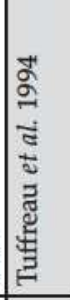 & 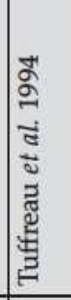 & 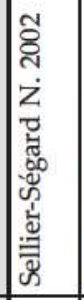 & 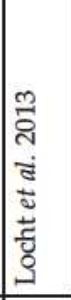 & 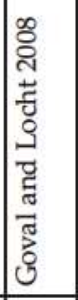 & 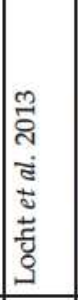 & 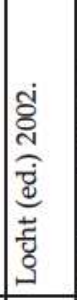 & 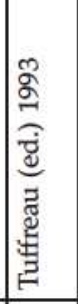 & 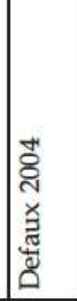 & 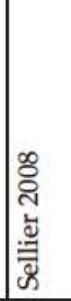 & 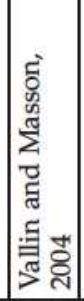 & 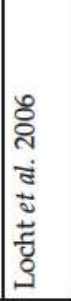 & 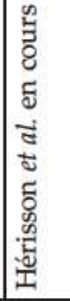 & 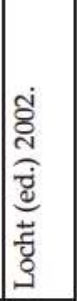 \\
\hline 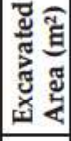 & F & I & $\vec{I}$ & $\underset{N}{\mathbb{N}}$ & $\stackrel{12}{=}$ & + & $\begin{array}{l} \\
\infty \\
\end{array}$ & $\underset{\sim}{\stackrel{\Xi}{*}}$ & 总 & \begin{tabular}{|l}
$\mathscr{\Xi}$ \\
î \\
\end{tabular} & \begin{tabular}{|c}
$\widetilde{N}$ \\
तె
\end{tabular} & ff & ले & ก̃ & \begin{tabular}{|l} 
总 \\
离
\end{tabular} & 总 & สี & $\begin{array}{l}\mathscr{8} \\
\infty \\
\end{array}$ & i & $\begin{array}{c}\infty \\
m \\
m\end{array}$ & \begin{tabular}{|l}
$\mathscr{1}$ \\
in \\
\end{tabular} & 8 & ঙ্লি & \&ू & 疋 \\
\hline 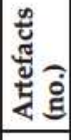 & $\stackrel{0}{\circ}$ & ले & న్ల & $\overline{\text { ส }}$ & 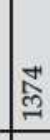 & + & 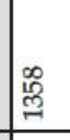 & $\infty$ & $\stackrel{m}{\stackrel{m}{-1}}$ & $\begin{array}{l}\text { त̂ } \\
\text { ते }\end{array}$ & $\Xi$ & گू & ह゙ & ชิ & $\not$ & I্ & 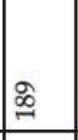 & 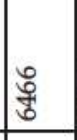 & 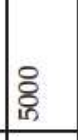 & 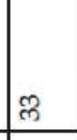 & 龸 & $\begin{array}{l}8 \\
\text { 离 } \\
\end{array}$ & ले & గై & $\ddot{m}$ \\
\hline صّ & 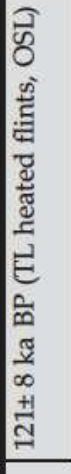 & & & 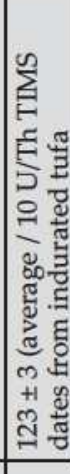 & & 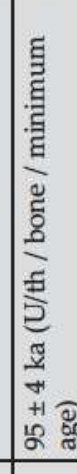 & & & 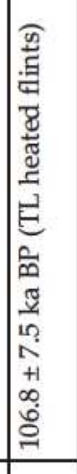 & 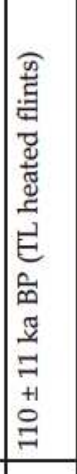 & 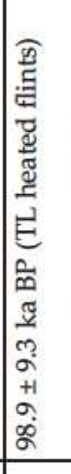 & 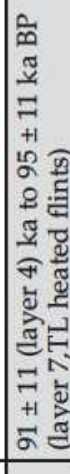 & 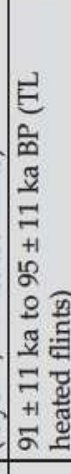 & & 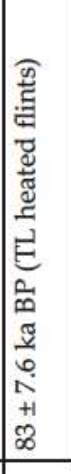 & & 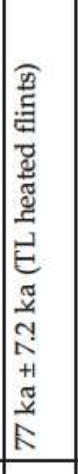 & & & & & & & & \\
\hline 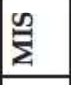 & เั & เก็ & เึ & เั & in & 픙 & I & เू & மூ & ம็ & ஜू & เั & ஜூ & ஜू & ஜ็ & ก็ & ก็ & เ็ & ก็ & ก็ & .๊ & เก็ & ก็ & เ็ & ก็ ฮี \\
\hline 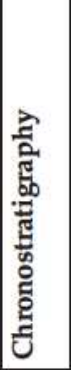 & 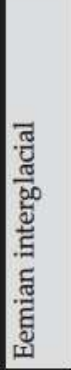 & 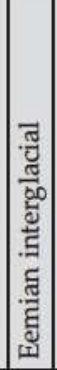 & 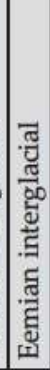 & 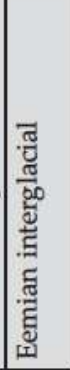 & 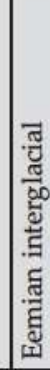 & 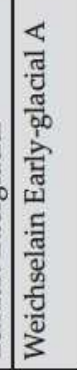 & 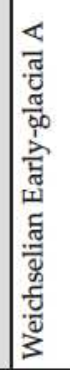 & 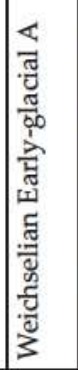 & 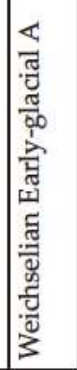 & 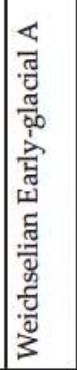 & 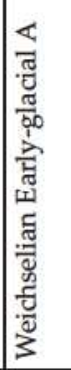 & 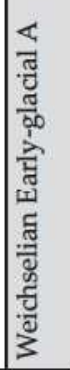 & 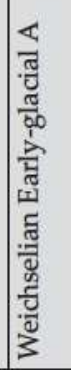 & 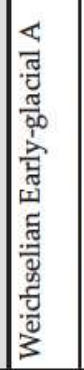 & 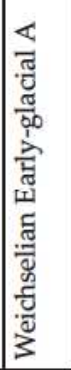 & 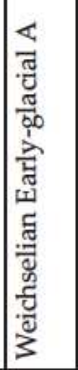 & 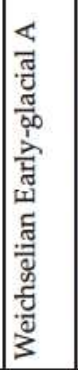 & 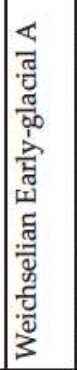 & 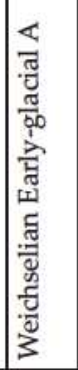 & 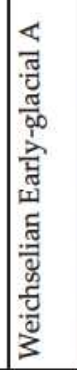 & 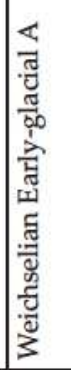 & 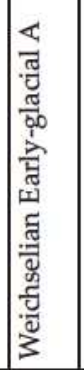 & 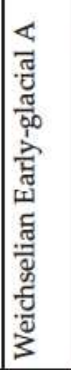 & 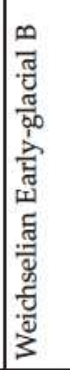 & 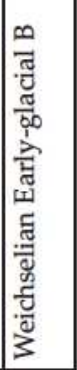 \\
\hline 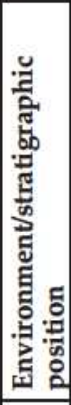 & 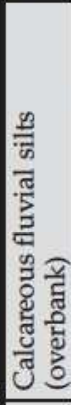 & 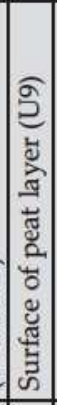 & 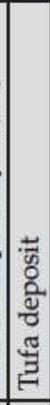 & 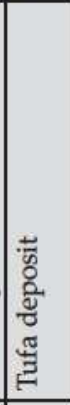 & 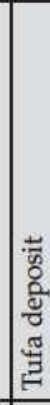 & 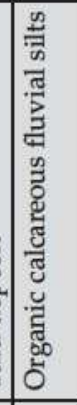 & 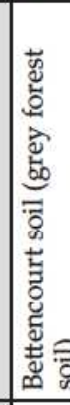 & 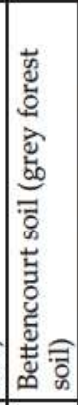 & 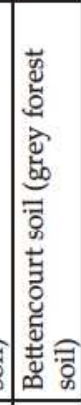 & 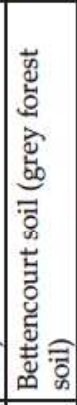 & 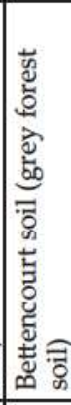 & 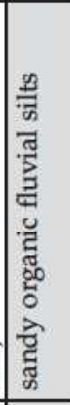 & 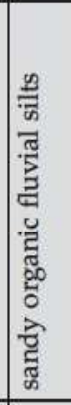 & 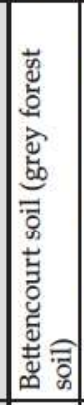 & 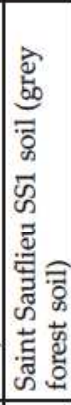 & 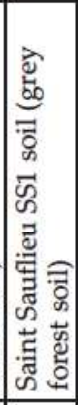 & 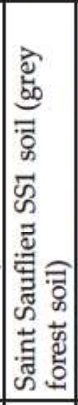 & 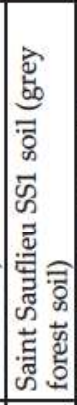 & 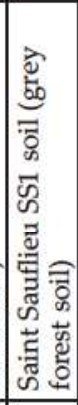 & 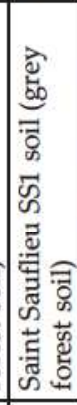 & 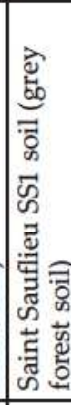 & 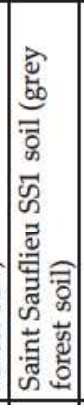 & 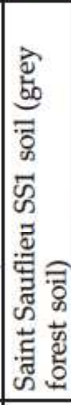 & 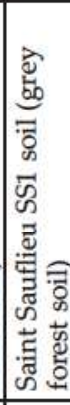 & 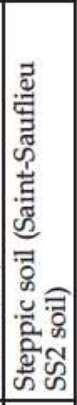 \\
\hline & 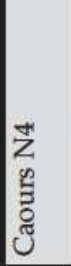 & 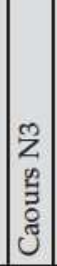 & 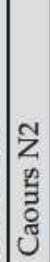 & \begin{tabular}{|l}
$\bar{z}$ \\
0 \\
$\tilde{\Xi}$ \\
$\tilde{\Xi}$ \\
$\tilde{J}$
\end{tabular} & 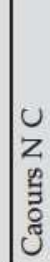 & 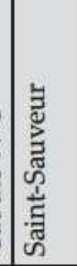 & 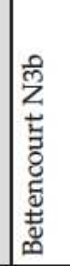 & 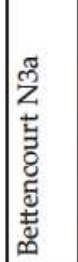 & 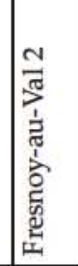 & 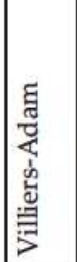 & 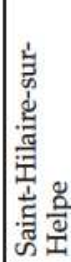 & 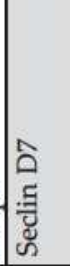 & 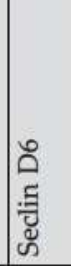 & 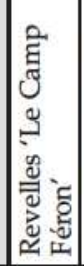 & 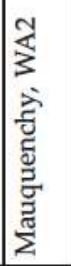 & 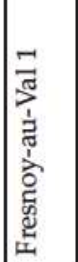 & 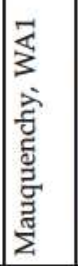 & 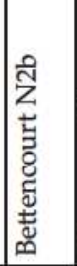 & 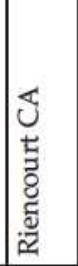 & 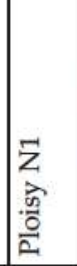 & 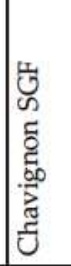 & 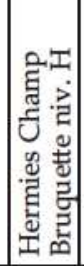 & 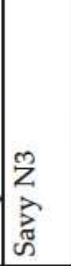 & 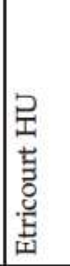 & 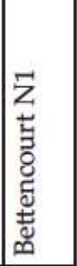 \\
\hline
\end{tabular}




\begin{tabular}{|c|c|c|c|c|c|c|c|c|c|c|c|c|c|c|c|c|c|c|c|c|c|c|c|}
\hline 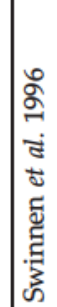 & | & 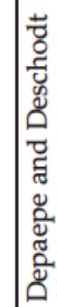 & 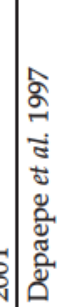 & 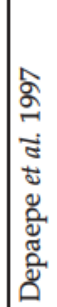 & 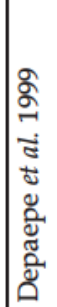 & 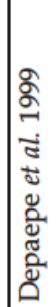 & 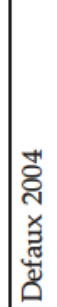 & 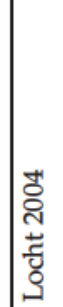 & 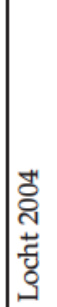 & 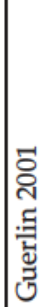 & 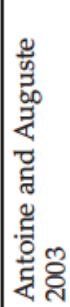 & 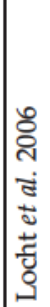 & 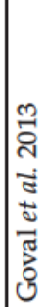 & 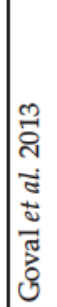 & 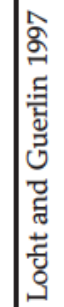 & 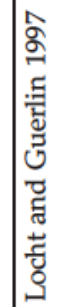 & 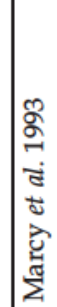 & 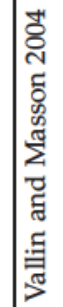 & 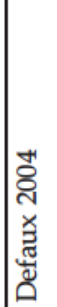 & 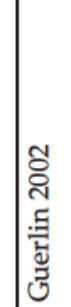 & 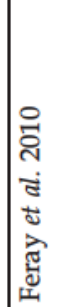 & 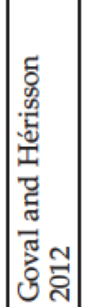 & 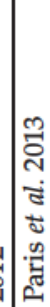 \\
\hline
\end{tabular}

倠

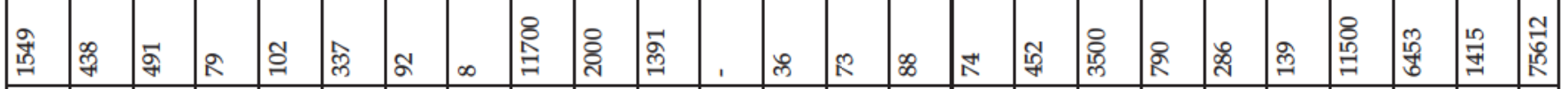

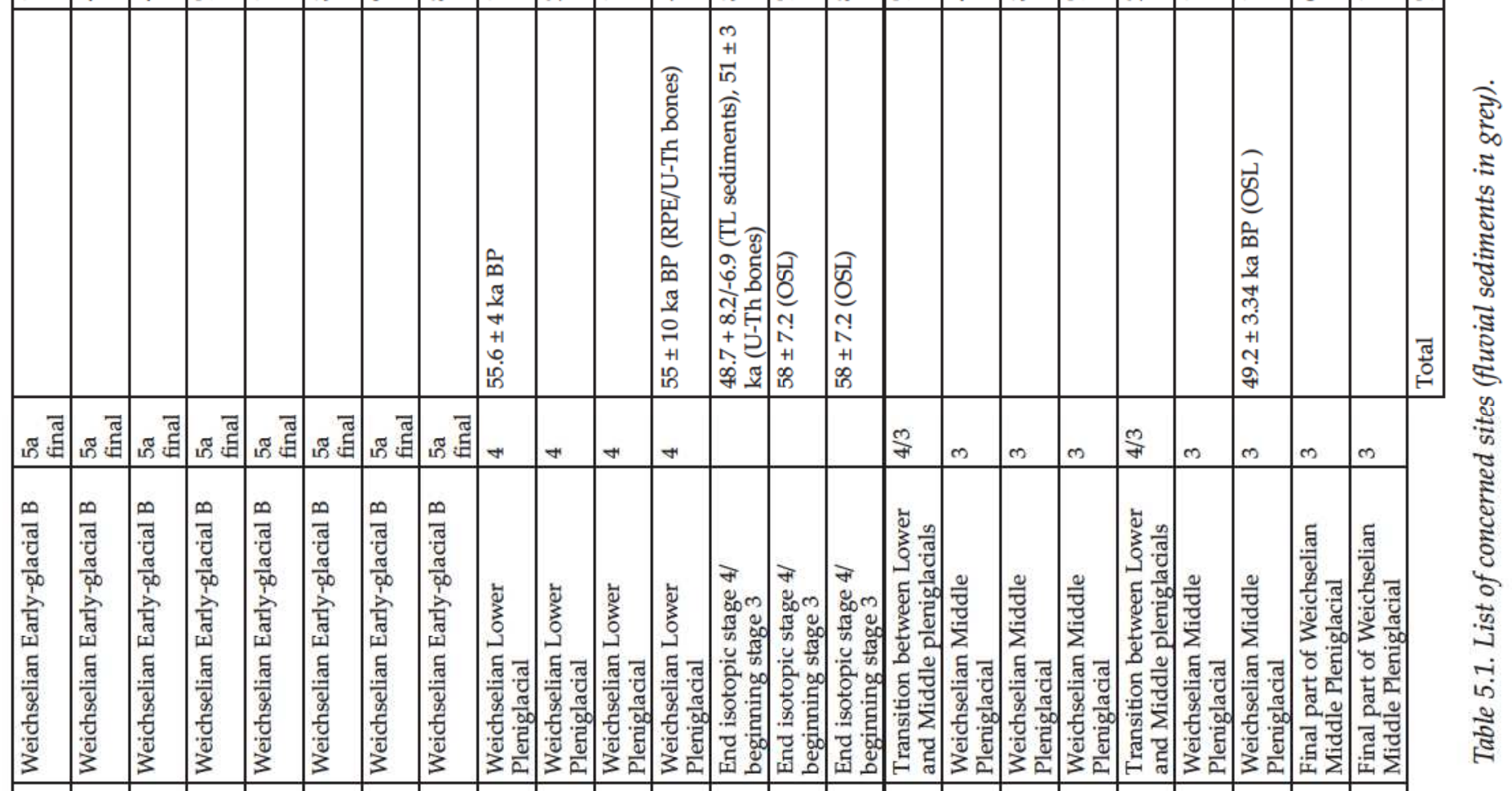

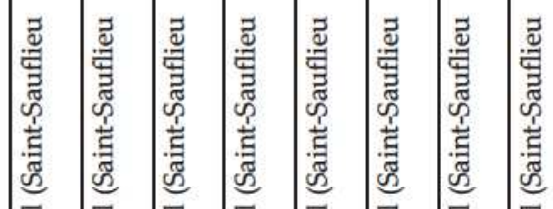

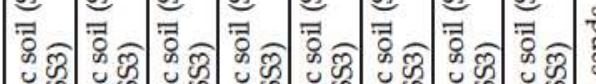

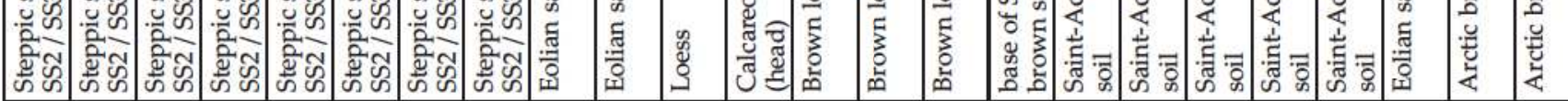

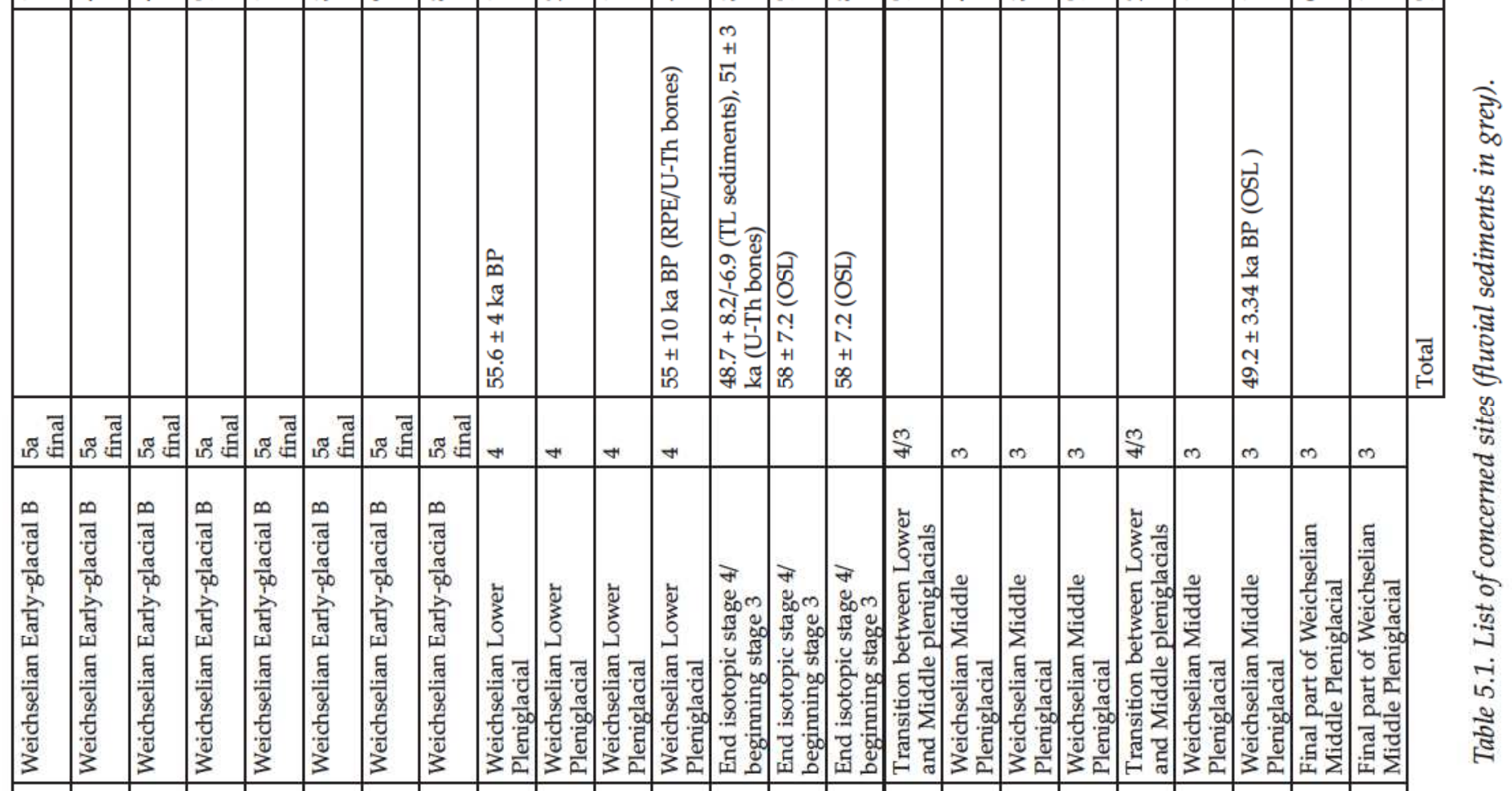

宫

.$\Xi$

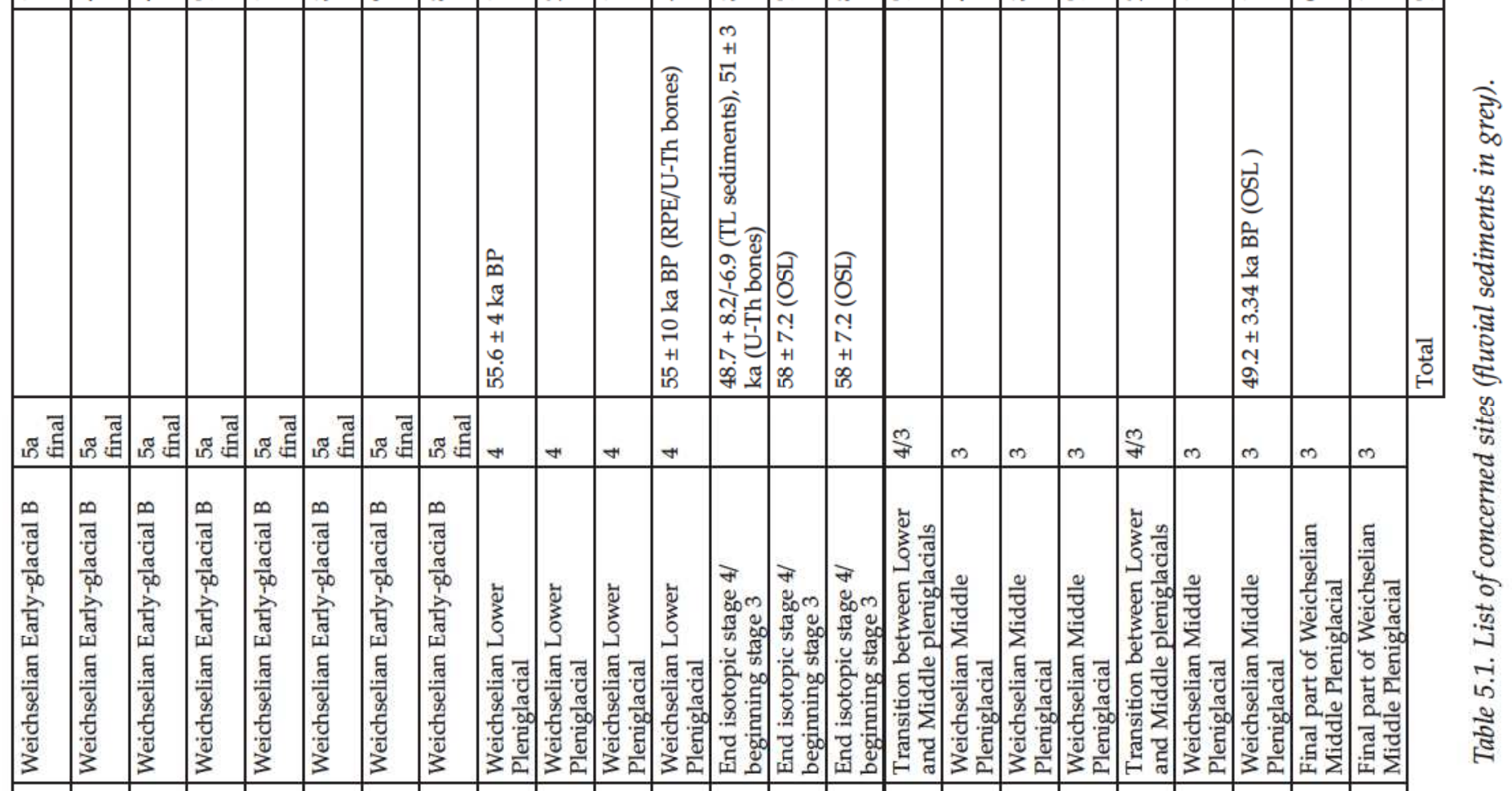

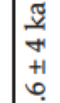

\begin{tabular}{|c|c|c|c|c|c|c|c|c|c|c|c|c|c|c|c|c|c|c|c|c|c|c|c|c|}
\hline & & & & & & & & 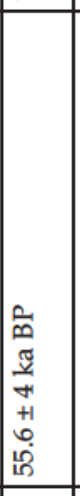 & & & 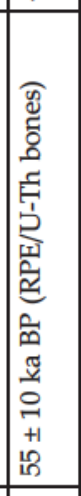 & 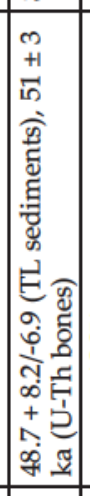 & 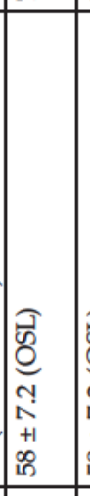 & 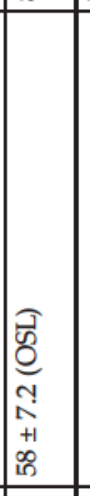 & & & & & & & 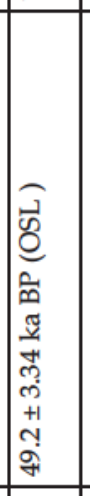 & & & 冚 \\
\hline ถ็ & ก๊ & ஸ็ $\frac{\pi}{0}$ & ก็ & in & in & 跑 & 活. & + & + & 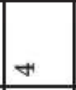 & H & & & & $\frac{1}{8}$ & $\infty$ & is & $\infty$ & $\stackrel{\Re}{\sharp}$ & $\infty$ & $m$ & $\infty$ & $\infty$ & \\
\hline 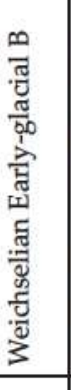 & 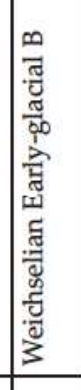 & 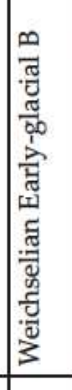 & 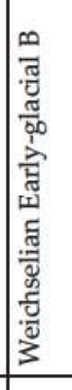 & 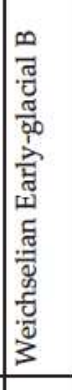 & 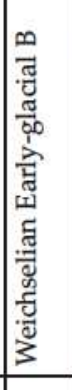 & 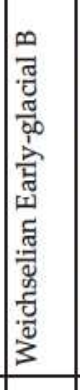 & 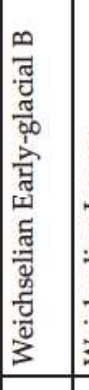 & 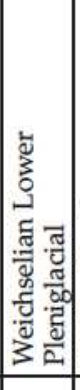 & 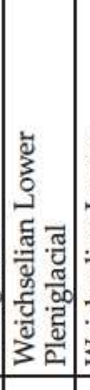 & 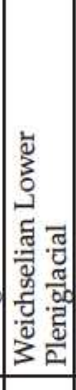 & 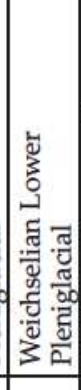 & 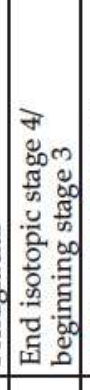 & 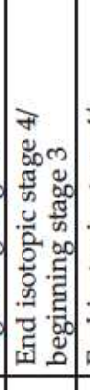 & 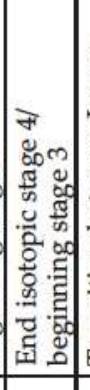 & 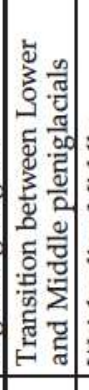 & 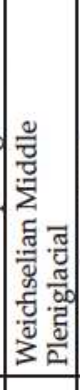 & 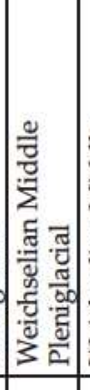 & 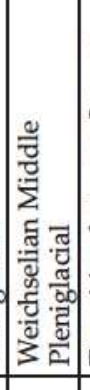 & 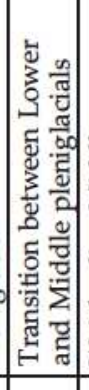 & 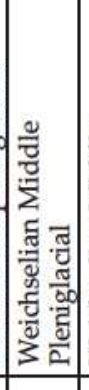 & 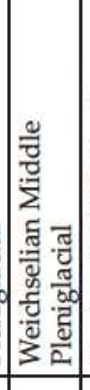 & 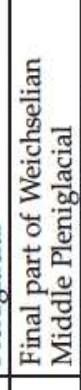 & 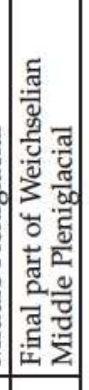 & \\
\hline
\end{tabular}

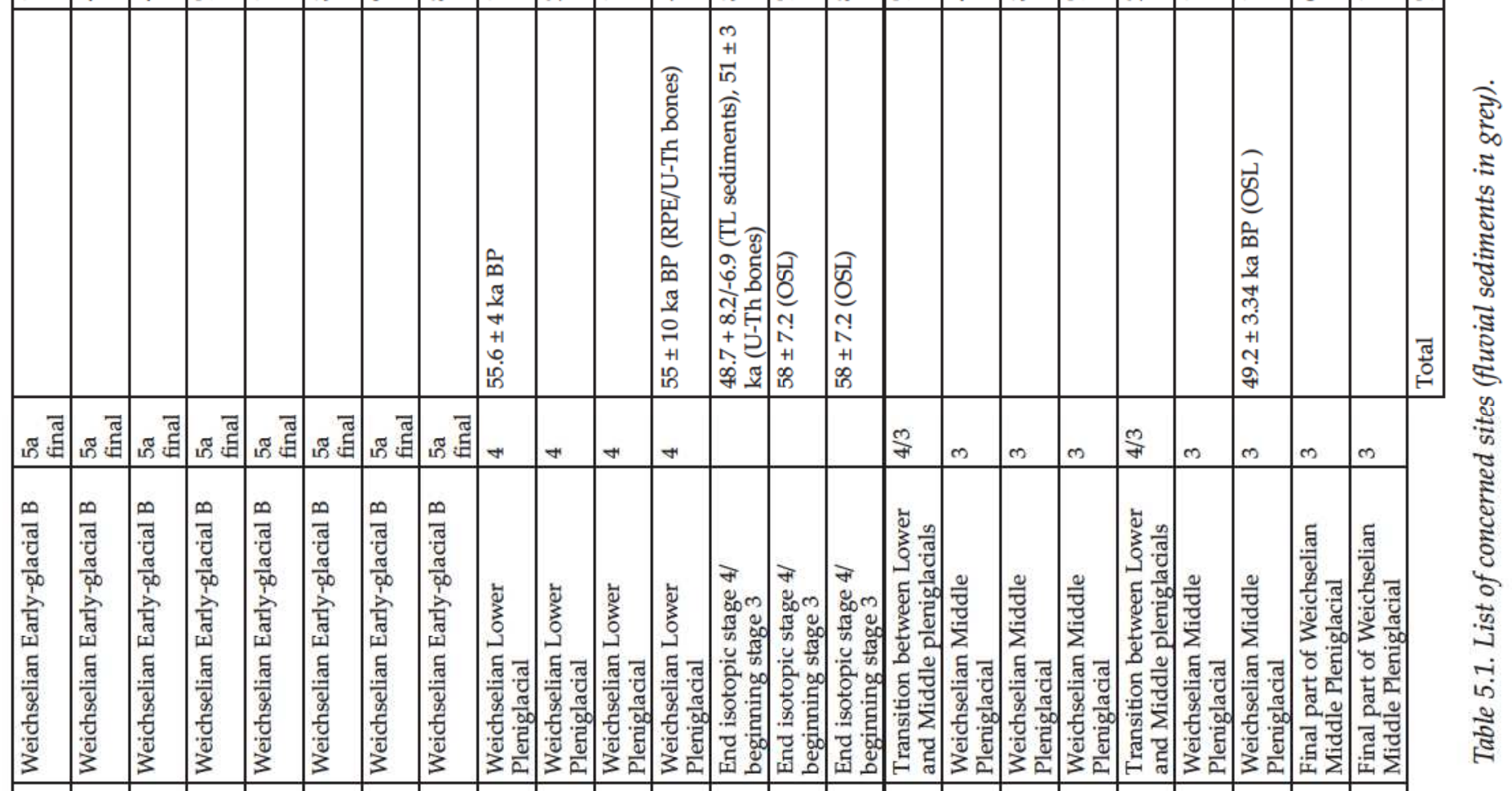

है

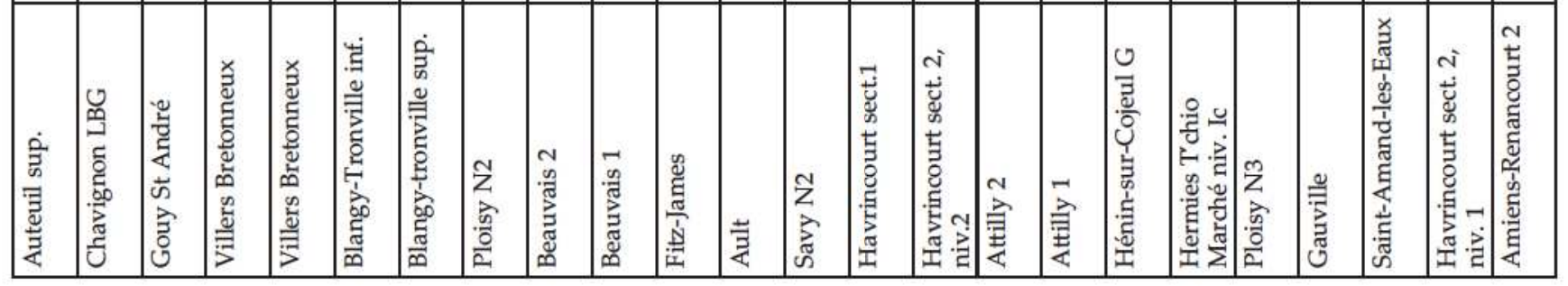




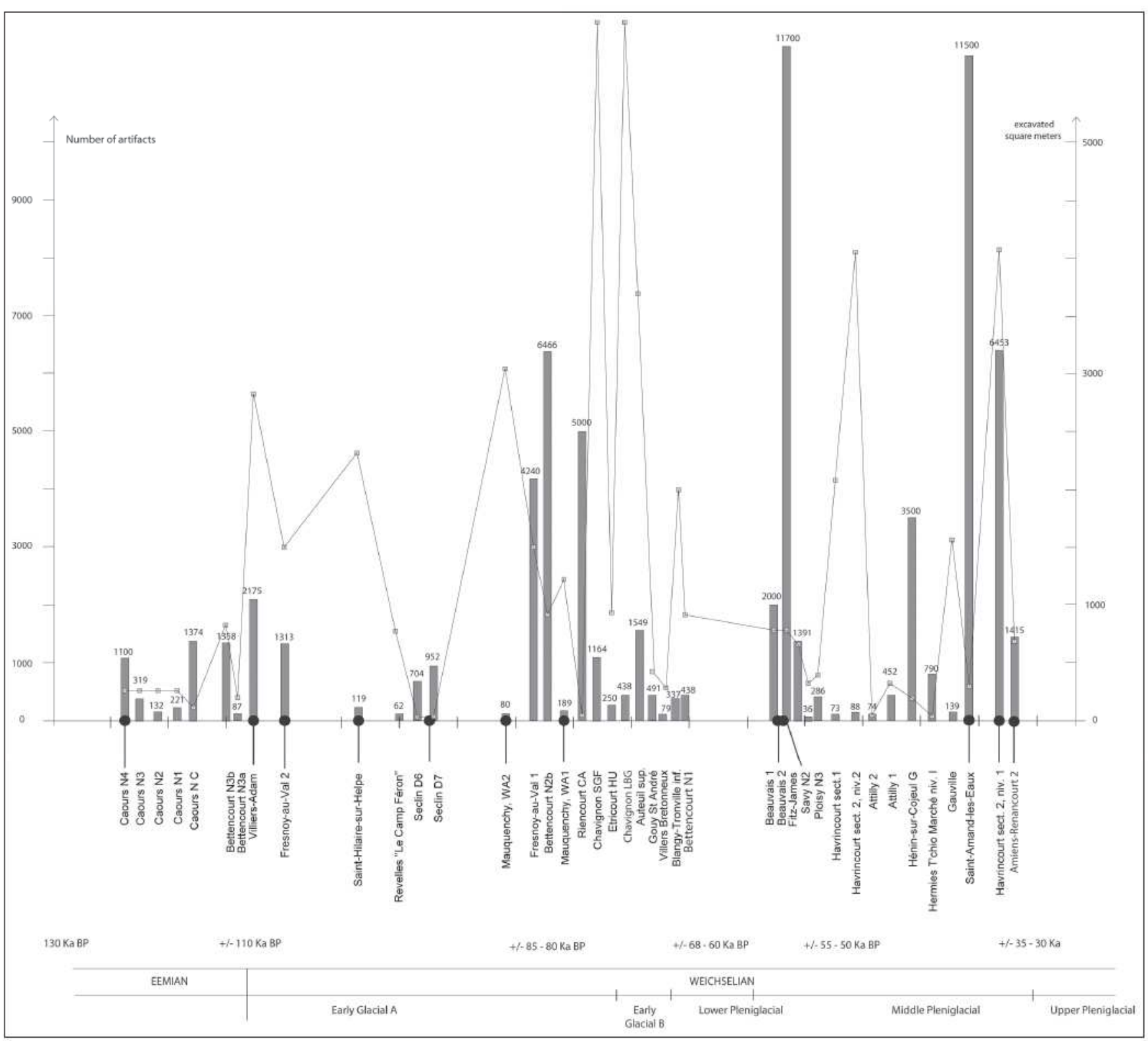

Figure 5.2. Excavated areas and number of artefacts.

2003 , etc.). The less abundant lithic series from the Middle Pleniglacial (MIS 3) show that these outcrops must still have been partly accessible (Gauville, Attilly, Havrincourt Sector 1) before being covered with Upper Pleniglacial loess.

\section{Climatic changes and lithic production systems}

Environmental changes do not seem to have had a direct impact on lithic reduction strategies. During the Eemian, the five occupation levels from Caours indicate discoidal reduction, sometimes associated with Levallois methods (Figure 5.4). Subsequently, during the early Weichselian, reduction strategies became more diversified and several systems coexist (blade core, Levallois, convergent unipolar and sometimes discoidal) with multiple production aims (blades, flakes, points, etc.). At the end of the Lower Pleniglacial, raw materials became accessible once again and lithic complexes are characterized by the exclusive presence of Levallois debitage. The site of Beauvais is an exception with two levels characterised by the sole presence of discoidal debitage (Locht 2004). Handaxe production is very poorly represented, thereby emphasising the specialised nature of sites with handaxes and mass handaxe production (Figure 5.4). 
main stratigraphic context
LF T Tuf
Bettencourt soil Bettencourt soil
(grey forest soil)
Saint-Sauflieu 1 soil Steppic
\begin{tabular}{|l|l|l|l|}
\hline SE Loess LB & Loess & $\begin{array}{c}\text { St Acheul } \\
\text { soil }\end{array}$ \\
\hline
\end{tabular}
Lower
brown soil

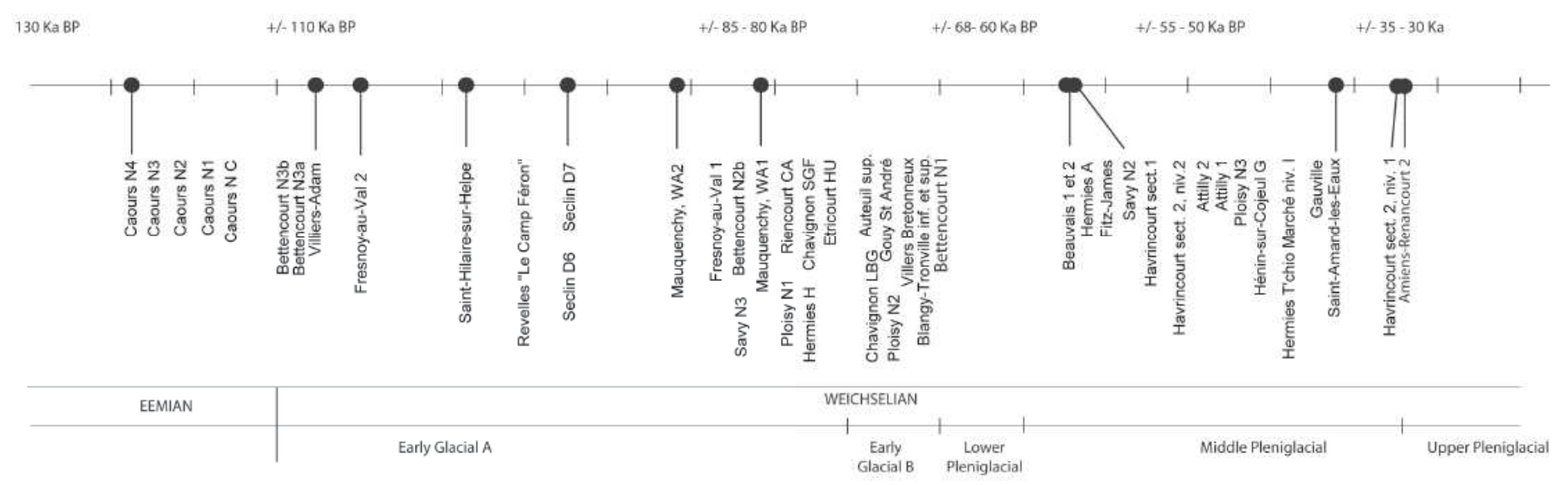

$\begin{array}{ll}- \text { precise dating (OSL, TL or C14) } & \text { LF Limon fluviatile } \\ \text { T } & \text { Tourbe } \\ \text { SE Sables éoliens } \\ \text { LB L Limon bruns }\end{array}$

Figure 5.3. Chronostratigraphical position of the archaeological levels.

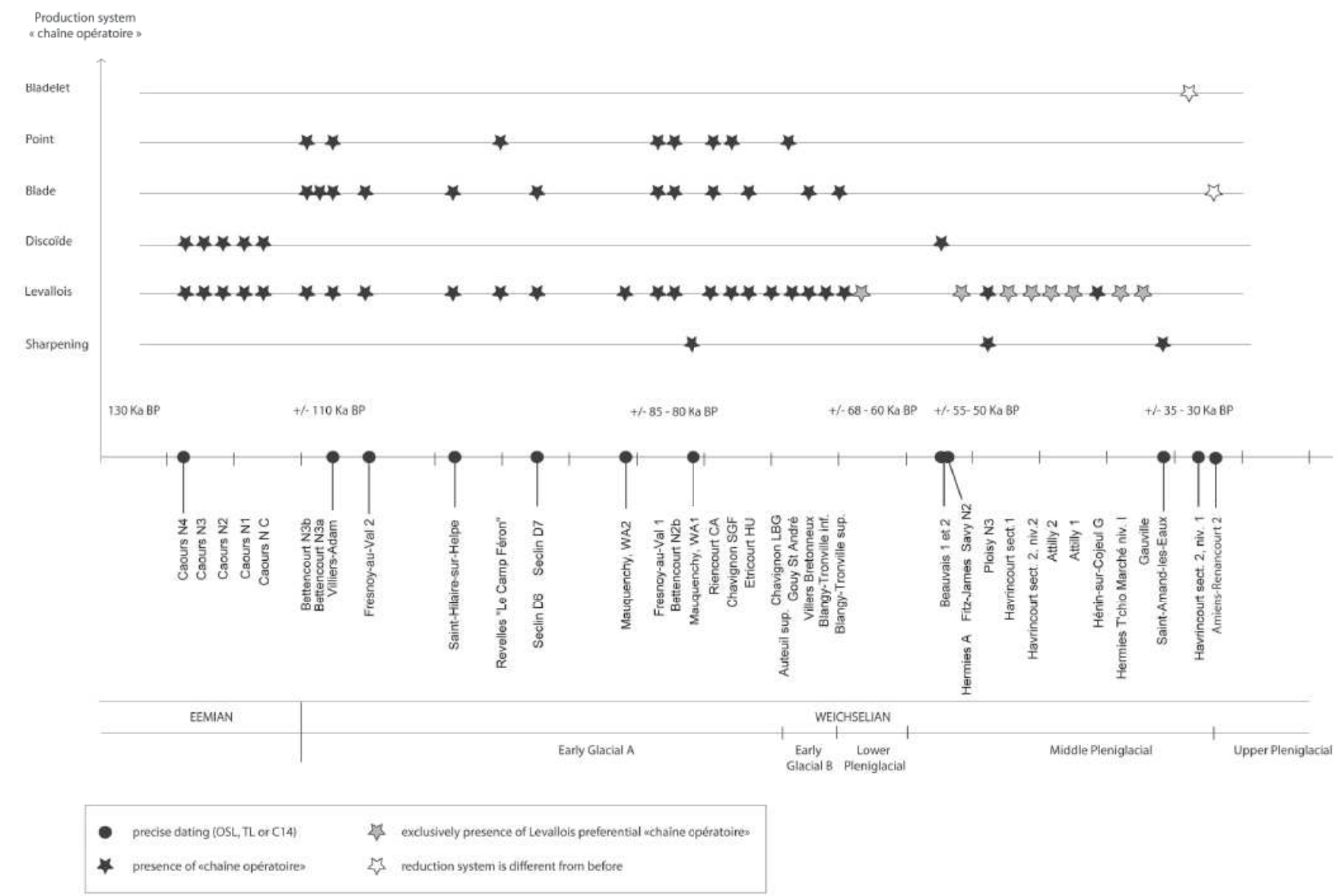

Figure 5.4. Characterisation of the main chaînes operratoires of the archaeological levels. 


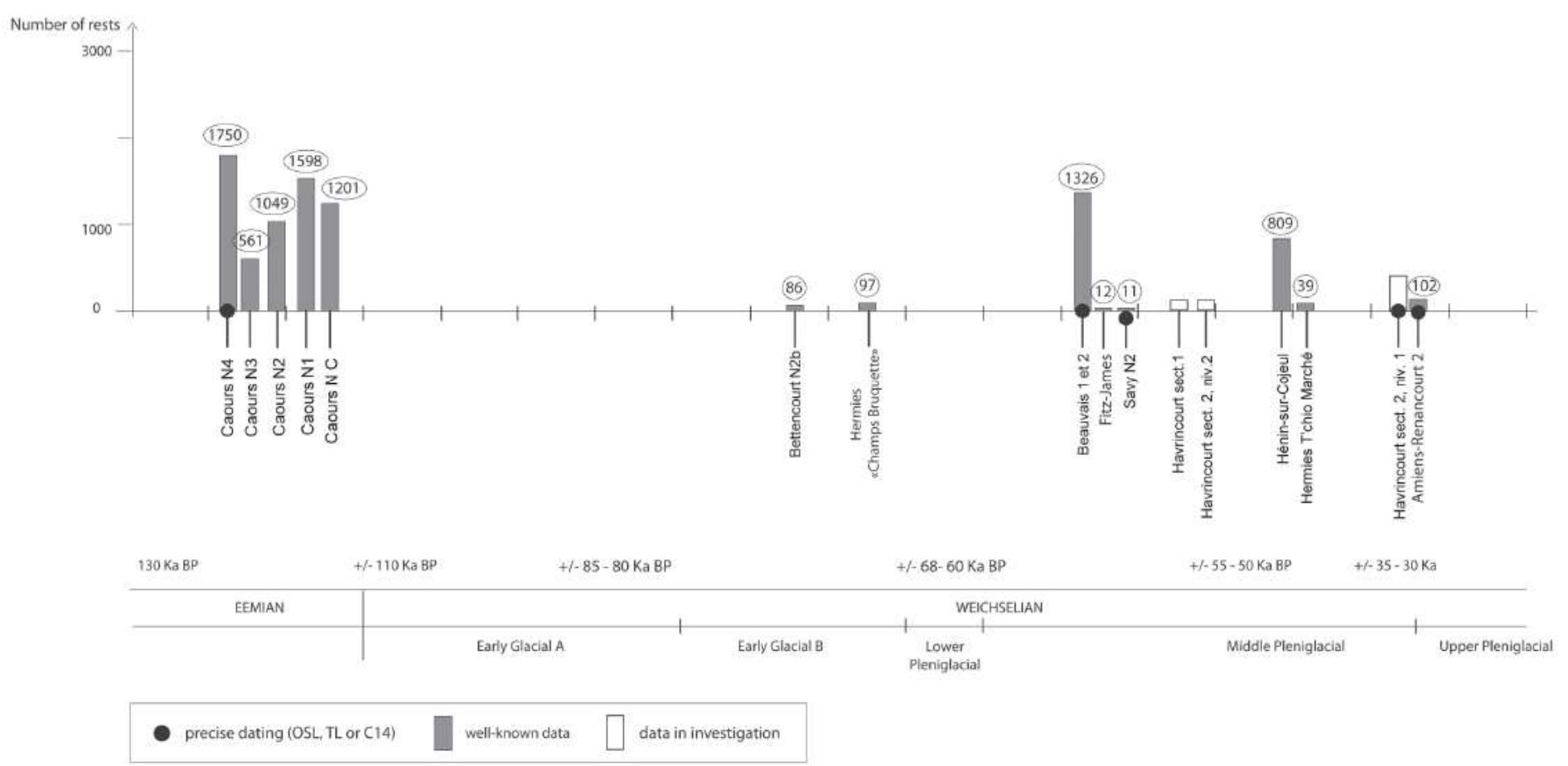

Figure 5.5. Number of preserved faunal remains.

\section{The impact of climatic changes on subsistence strategies}

Faunal data are less abundant, exclusively due to taphonomic reasons. Out of 47 occupations ascribed to the recent Middle Palaeolithic (MIS 5e to 3), only fourteen have yielded fauna (Figure 5.5). The two Early Upper Palaeolithic occupations (Havrincourt Sector 2, Level 1 and Renancourt 2) have also yielded abundant bone remains. During the Eemian, the fauna is dominated by cervids (red deer and fallow deer), but aurochs are also present, as shown by the five Caours levels. Generally speaking, the faunal series are then dominated by large herbivores from the beginning of the Weichselian glacial period, particularly horse and Steppe bison, like at Hermies or Héninsur-Cojeul (Auguste 2009). Woolly mammoth and woolly rhinoceros are also present, though much less abundant. However, mammoth plays a central role at certain sites, such as Ault. During phases of climatic deterioration, reindeer is present in the spectrum, e.g. at Beauvais and Havrincourt. All these occupations are mainly characterised by the hunting of large herbivores, although are occasionally oriented towards smaller animals such as reindeer. The butchery activities that have been identified at these sites attest to the removal of meat, occasionally accompanied by the breakage of long bones, indicating diversified subsistence strategies that may be linked to the seasonality of occupation. In all of the sites younger than the Eemian, the environment is always open and dominated by grasslands during clement phases, such as at Bettencourt-Saint-Ouen, and steppe during cold phases (Auguste 2012).

\section{Discussion}

Environmental changes had an impact on human access to flint, which was the main raw material during the Palaeolithic occupation of the north of France. Generally speaking, access to deposits was hampered by the presence of forests, which stimulated the procurement of flint from secondary sources. The flint nodules that were exploited are, therefore, relatively small in size and variable in quality. During the Lower Pleniglacial, slopes were eroded and flint bars became easily accessible on the chalky talus. Correspondingly, good-quality raw material was available in larger sized modules, propitious to the production of large preferential Levallois flakes.

Climatic change does not seem to have influenced the choice of lithic reduction strategies. The case of Bettencourt-Saint-Ouen is evocative and shows the persistence of similar needs (chaîne opératoires oriented towards the production of points, blades and flakes using identical technical schemas) for more than 40,000 years in the same area, across differing environments (forest at the base and steppe towards the top). This stability 
in lithic reduction strategies underlines the importance of cultural traditions in the face of environmental modifications (Locht 2002; Antoine et al. 2003). Recurring occupations at the northern French sites suggests some kind of management of territory, as well as the transmission of technical traditions throughout time.

Climatic modifications do not appear to influence Neanderthal subsistence strategies other than varying their main herbivorous prey taxa. Similar behaviour can be observed at sites characterised by contrasting environments, e.g. interglacial (Caours) and Pleniglacial (Beauvais) where subsistence strategies were identical. Hunting preferentially targeted small or medium sized herbivores, although larger species are present. Transportable animal carcasses, such as those of red deer, fallow deer, and reindeer, were brought back whole to butchery sites, while larger animals (e.g. elephant, aurochs, mammoth, and rhinoceros) seem to have been butchered at the kill site and brought back in quarters. At both sites, tools produced by discoidal reduction were mainly used for carcass processing. At other sites, however, large herbivores dominate, highlighting different subsistence strategies.

The occupations of both Neanderthals and the first anatomically humans in the north of France are correlated with phases of climatic amelioration. Both groups displayed the ability to adapt to their environment and their behaviour does not seem to have been very different. Thus, the animal and lithic procurement strategies demonstrate that subsistence behaviour and anticipation were similar during the Middle and Upper Palaeolithic (Locht 2004).

\section{Bibliography}

Antoine P., Munaut A.-V. and Somme J. (1994) Réponse des environnements à l'évolution climatique du Début Glaciaire weichselien: données de la France du Nord Ouest. Quaternaire 5, 151-56.

Antoine P., Munaut A.-V., van Kolschoten T. and Limondin N. (1995) Une occupation du Paléolithique moyen en contexte fluviatile dans la séquence de la très basse terrasse de la Somme à Saint-Sauveur (Somme). Bulletin de la Société Préhistorique Française 92(2), 201-12.

Antoine P. and Auguste P. (2003) La paléofalaise et le site paléolithique d'Ault-Onival (Somme): données anciennes et nouvelles recherches. In: J.-M. Hoeblich (ed.) Les falaises de Picardie, état des lieux, enjeux, actions, Actes du Colloque -Amiens, 6-7 Avril 2001, pp. 39-46. Université de Picardie: Association pour le littoral picard et la Baie de Somme.

Antoine P., Limondin-Lozouet N., Auguste P., Lamotte A., Bahain J.-J., Falgueres C., Laurent M., Coudret P., Locht J.-L., Depaepe P., Fagnart J.-P., Fontugne M., Hatte C., Mercier N., Frechen M., Moigne A.-M., Munaut A.-V., Ponel P. and Rousseau D.-D. (2003) Paléoenvironnements pléistocènes et peuplements paléolithiques dans le bassin de la Somme (nord de la France). Bulletin de la Société préhistorique Française 100(1), 5-28.

Antoine P, Limondin-Lozouet N., Auguste P., Locht J.-L., Galheb B., Reyss J. L, Escudé E., Carbonel P., Mercier N., Bahain J.-J., Falguères C. and Voinchet (2006). Le site de Caours (Somme / France): mise en évidence d'une séquence de tuf contemporaine du dernier interglaciaire (Eemien) et d'un gisement paléolithique associé, Quaternaire 17(4), 281-320.

Antoine, P., Bahain, J.-J., Auguste, P., Fagnart, J.-P., LimondinLozouet, N. and Locht, J.-L. (2011a) Quaternaire et Préhistoire dans la vallée de la Somme: 150 ans d'histoire commune. In: A. Hurel and N. Coye (eds) Dans l'épaisseur du temps Archéologues et géologues inventent la préhistoire, pp. 341-79. Paris: Publication du MNHN.

Antoine, P., Auguste, P., Bahain, J.-J. and Louguet, S. (2011b) Datation et reconstitution paléoenvironnementale d'un site paléolithique moyen submergé en Manche Est: Ault-Onival (Somme, France). Quaternaire 22, 221-33.

Auguste P. (2009) Évolution des peuplements mammaliens en Europe du Nord-Ouest durant le Pléistocène moyen et supérieur. Le cas de la France septentrionale. Quaternaire, 20(4), 527-50.

Auguste P. (2012) L'homme et l'animal au Pléistocène en France septentrionale. Un quart de siècle de recherches paléontologiques et archéozoologiques dans le nord de la France. Université Lille: Habilitation à Diriger des Recherches.

Defaux F. (2004) Ploisy « Le Bras de Fer ». Amiens: Rapport de fouille, INRAP, Service Régional de l'Archéologie de Picardie.

Depaepe P. and Deschodt L. (2001) Le site de Gouy-SaintAndré 'Le Savigny'. In: A. Tuffreau (ed.) L'Acheuléen dans la vallée de la Somme et Paléolithique moyen dans le Nord de la France: données récentes, pp. 185-98. Lille: Université des Sciences et Technologies de Lille-Flandres-Artois, Publications du CERP 6.

Depaepe P., Locht J.-L. and Swinnen C. (1997) Découverte de sites du Paléolithique moyen sur le tracé d'un gazoduc en Picardie (France). Notae Praehistoricae 17, 25-38.

Depaepe P., Antoine P., Guerlin O. and Swinnen C. (1999) Le gisement paléolithique moyen de Blangy-Tronville (Somme). Revue Archéologique de Picardie 3-4, 3-21.

Feray P., Deschodt L., Lantoine J. and Schwenninger J.-L. (2010) Le site paléolithique moustérien de tradition acheuléenne (MTA) de Saint-Amand-les-Eaux (nord de la France). Résultats préliminaires. Poster presented at Pré-actes du colloque Q7 'Bio-géosystèmes continentaux quaternaires: variabilité climatique et anthropisation', Besançon 17-19 Février 2010.

Goval E. and Locht J.-L. (2009) Remontages, systèmes techniques et répartitions spatiales dans l'analyse du site weichselien ancien de Fresnoy-au-Val (Somme, France). Bulletin de la Société préhistorique Française 106(4), 653-78.

Goval E. and Herisson D. (2012) Découverte inédite de trois occupations du Pléniglaciaire moyen du Weichselien à Havrincourt 'Les Bosquets' (Pas-de-Calais, France). Bulletin de la Société préhistorique Française 109(2), 331-58

Goval E., Herisson D., Antoine P., Auguste P., Bahain J.-J., Claud E., Coutard S., Font C., Guerrin G., Hulin G., Jamet G., Moine O., Prilaux G., Schmidt E. and Valladas H. (2013) 
Des derniers Néandertaliens aux premiers Homo Sapiens: les occupations paléolithiques d'Havrincourt 'Les Bosquets' (Pasde-Calais). Service Régional de l'Archéologie Pas-de-Calais: Rapport de fouille du Canal Seine-Nord Europe.

Guerlin O. (2001) Les industries du Paléolithiques de FitzJames (Oise). Mémoire de Maîtrise. Dijon: Université de Bourgogne.

Guerlin O. (2002) Gauville. Rapport d'évaluation archéologique. Amiens: INRAP, Service Régional de l'Archéologie de Picardie.

Guthrie D. R. (1982) Mammals of the Mammoth Steppe as Paleoenvironmental Indicators. In: D. M. Hopkins, J. V. Matthews Jr, C. E. Schweger, and S. Young (eds) Paleoecology of Beringia, pp. 307-29. New York: Academic Press.

Herisson D., Goval E., Coutard S., Locht J.-L., Antoine P. and Chantreau Y. (in press) Le gisement paléolithique inférieur et moyen d'Etricourt-Manancourt (Somme). Amiens: Canal Seine-Nord-Europe, fouille 49.2, INRAP, Service Régional de l'Archéologie de Picardie.

Lantoine J. and Feray P. (2006) Saint-Hilaire-sur-Helpe. Carrière des Ardennes, Extension. Bilan scientifique du Nord-Pas-deCalai. Nord-Pas-de-Calais: Direction Régional des Affaires Culturelles.

Locht J.-L. (2002) Bettencourt-Saint-Ouen: cinq occupations paléolithiques au début de la dernière glaciation. Document d'Archéologie Française 90, 169.

Locht J.-L. (2004) Le Gisement paléolithique moyen de Beauvais (Oise). Contribution à la connaissance des modalités de subsistance des chasseurs de renne du Pléniglaciaire inférieur $d u$ Weichselien. Unpublished $\mathrm{PhD}$ Thesis. Université Lille-I.

Locht J.-L. and Guerlin O. (1997) Le gisement paléolithique moyen I du Bois de la Bocquillière, à Attilly (Aisne). Amiens: Rapport d'évaluation, AFAN, Service Régional d'Archéologie de Picardie.

Locht J.-L., Antoine P., Bahain J.-J., Limondin-Lozouet N., Gauthier A., Debenham N., Frechen M., Dwrila G., Raymond P., Rousseau D. D., Hatté C., Haesaerts P. and Metsdagh H. (2003). Le gisement paléolithique moyen et les séquences pléistocènes de Villiers-Adam (Val d'Oise, France): Chronostratigraphie, environnement et implantations humaines. Gallia Préhistoire 45, 1-111.

Locht J.-L., Antoine P., Auguste P., Bahain J.-J., Debenham N., Falguères C., Farkh S. and Tissoux H. (2006) La séquence loessique pléistocène supérieur de Savy (Aisne, France): stratigraphie, datations et occupations paléolithiques. Quaternaire 17(3), 269-75.

Locht J.-L., Goval E. and Antoine P. (2010) Reconstructing
Middle Palaeolithic Hominids Behaviour during OIS 5 in Northern France. In: N. Conard and A. Delanges (eds) Settlement Dynamics of the Middle Palaeolithic and Middle Stone Age, Volume III, pp. 329-55. Tübingen: Kerns Verlag.

Locht J.-L., Sellier N., Antoine P., Koehler H. and Debenham N. (2013) Mauquenchy (Seine-Maritime, France): mise en évidence de deux niveaux d'occupation paléolithique dans un sol gris forestier daté du SIM 5a (Début Glaciaire weichselien). Quaternaire 24(3), 247-257.

Marcy J.-L., Auguste P., Fontugne M., Munaut A.-V., van Vliet-Lanoë B. (1993) Le gisement moustérien d'Héninsur-Cojeul (Pas-de-Calais). Bulletin de la Société préhistorique Française 90(4), 251-56.

Paris C., Fagnart J.-P. and Coudret P. (2013) Du Gravettien final dans le Nord de la France? Nouvelles données à Amiens-Renancourt. Bulletin de la Société préhistorique Française, 110(1), 123-26.

Roebroeks W. and Speleers B. (2002) Last Interglacial (Eemian) Occupation of the North European Plain and Adjacent Areas. In: A. Tuffreau and W. Roebroeks (eds) Le Dernier Interglaciaire et les occupations humaines du Paléolithique moyen, pp. 31-39. Publication du CERP, Lille 8.

Sellier-Segard N. (2002) Revelles 'Camp Féron'. Amiens: Rapport d'évaluation A29. Ouest. INRAP, SRA Picardie. Bulletin de la Société préhistorique Française

Sellier N. (2008) Chavignon. Déviation de la RN 2. Amiens: Rapport de fouille, INRAP, SRA Picardie.

Swinnen C., Locht J.-L. and Antoine P. (1996) Le gisement moustérien d'Auteuil (Oise). Bulletin de la Société préhistorique Française 93(2), 173-81.

Teheux E. (2000) Le gisement paléolithique moyen de 'La Minette' à Fitz-James. Archéopages 1, 30-37.

Tuffreau A. (1993) Riencourt-les-Bapaume (Pas-de-Calais). Un gisement du Paléolithique moyen. Documents d'Archéologie Française 37. Paris: Publishing House of Human Sciences.

Tuffreau A., Revillion S., Somme J. and van Vliet-Lanoë B. (1994) Le gisement paléolithique moyen de Seclin (Nord). Bulletin de la Société préhistorique Française, 91(1), 23-46.

Vallin L. and Masson B. (2004) Behaviour towards Lithic Production during the Middle Palaeolithic: Examples from Hermies le Champ Bruquette and Hermies le Tio Marché (Pas-de-Calais, France). In: E. A. Walker, F. Wenban-Smith, and F. Healy (eds) Lithics in action. Papers from the conference Lithic Studies in the year 2000, Lithic Studies Society Occasional Paper 8, pp. 5-25. Oxford: Oxbow Books. 